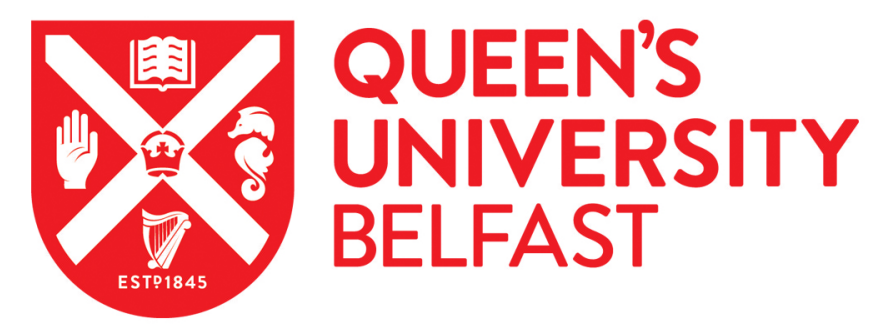

\title{
Relaxing DRAM Refresh Rate through Access Pattern Scheduling: A Case Study on Stencil-based Algorithms
}

Tovletoglou, K., Nikolopoulos, D. S., \& Karakonstantis, G. (2017). Relaxing DRAM Refresh Rate through Access Pattern Scheduling: A Case Study on Stencil-based Algorithms. In 23rd IEEE International Symposium on OnLine Testing and Robust System Design 2017: Proceedings (pp. 1-6)

https://doi.org/10.1109/IOLTS.2017.8046197

Published in:

23rd IEEE International Symposium on On-Line Testing and Robust System Design 2017: Proceedings

\section{Document Version:}

Peer reviewed version

Queen's University Belfast - Research Portal:

Link to publication record in Queen's University Belfast Research Portal

Publisher rights

(c) 2017 IEEE. This work is made available online in accordance with the publisher's policies. Please refer to any applicable terms of use of the publisher.

\section{General rights}

Copyright for the publications made accessible via the Queen's University Belfast Research Portal is retained by the author(s) and / or other copyright owners and it is a condition of accessing these publications that users recognise and abide by the legal requirements associated with these rights.

Take down policy

The Research Portal is Queen's institutional repository that provides access to Queen's research output. Every effort has been made to ensure that content in the Research Portal does not infringe any person's rights, or applicable UK laws. If you discover content in the Research Portal that you believe breaches copyright or violates any law, please contact openaccess@qub.ac.uk. 


\section{Relaxing DRAM Refresh Rate through Access Pattern Scheduling: A Case Study on Stencil-based Algorithms}

\author{
Konstantinos Tovletoglou \\ EEECS, Queen's University Belfast \\ Email: ktovletoglou01@qub.ac.uk
}

\author{
Dimitrios S. Nikolopoulos \\ EEECS, Queen's University Belfast \\ Email: d.nikolopoulos@qub.ac.uk
}

\author{
Georgios Karakonstantis \\ EEECS, Queen's University Belfast \\ Email: g.karakonstantis@qub.ac.uk
}

\begin{abstract}
The main memory in today's systems is based on DRAMs, which may offer low cost and high density storage for large amounts of data but it comes with a main drawback; DRAM cells need to be refreshed frequently for retaining the stored data. The refresh rate in modern DRAMs is set based on the worst-case retention time without considering access statistics, thereby resulting in very frequent refresh operations. Such high refresh rate leads eventually to large power and performance overheads, which are increasing with higher DRAM densities. However, such high refresh rates may not even required due to extremely low probability of the actual occurrence of the assumed worst-case scenarios, or due to the implicit refresh operation that occur during every memory access, a feature that has not been yet been studied in depth. In this paper, we enhance the state-of-the-art by systematically exploiting the implicit refresh of memory access for relaxing the refresh rate, while minimizing the resulting memory errors. This is achieved by modifying the algorithmic parameters that influence the access patterns such that all stored data are being touched within a target time interval that is necessary for meeting a target error rate. The proposed method is applied to stencil-based algorithms which represent a wide class of algorithms used in numerical analysis, image processing and cellular automata applications. The efficacy of the proposed method is demonstrated on an off-the-shelf server running a fully fledged Linux OS and results show that it is even possible to completely disable DRAM refresh with minor quality loss.
\end{abstract}

\section{INTRODUCTION}

The ever increasing need for higher memory capacity is driving the aggressive scaling of Dynamic Random-Access Memory (DRAM), which is an essential component of all computing systems. However, aggressive DRAM scaling is hampered by the need of periodic refresh operations to retain the stored data, the frequency of which is conventionally being determined by the worst-case retention time of the most leaky cell. Such an approach might help to achieve error free storage, but its viability is in doubt due to the large waste of power and throughput that may incur reaching up-to $25-40 \%$ and $15-30 \%$ respectively, in future $32 \mathrm{~Gb}-64 \mathrm{~Gb}$ densities [1].

To address such an alarming challenge, many recent studies have shown that the retention time of DRAM cells varies a lot and most of the cells do not require as frequent refresh as the conventional paradigm dictates [2]. Based on such an observation recent approaches [1], [3] proposed to group rows into different retention bins and apply a high refresh rate only for rows of low retention times. However, such multiraterefresh techniques not only require costly and intrusive hardware modifications but also they do not take into account the fact that in practice the retention time of cells changes over time [4], thus rendering error-free storage impossible.
A limited number of studies [5], [6] have also attempted to leverage the inherent error-resilience of various applications to relax DRAM refresh rates. However, these studies neglect to identify and make use of the inherent ability of applications to refresh memory by accessing their own data, a property that we call Refresh-by-Access (RefA). We set the exploitation of RefA as the main target of this paper.

We observe that in current DRRx technologies, the refresh operation and memory accesses are mutually exclusive to each other. This means that no row/chip within a rank are allowed to be accessed while the specific rank is being refreshed. This does not only cause a performance penalty, but also prevents the exploitation of the implicit refresh incurred with each memory read, which can in turn help the system relax the conventional Auto-Refresh $(A R)$ process. Recent works have tried to exploit row access locality [7] or read operations [8] for relaxing refresh. Such methods have demonstrated the potential benefits of exploiting RefA but have been impractical and evaluated only in simulation, since they require intrusive modifications of the memory controller.

In this paper, we aim at exploiting systematically the RefA property for facilitating aggressive refresh relaxation. This is achieved by developing non-intrusive methods based on the intelligent selection of application parameters that lead to an adequate frequency of memory accesses while satisfying performance and quality constraints. The proposed methods are applied on popular stencil-based algorithms and are evaluated on a real server-grade system stack. Our contributions are summarized as follows:

- We introduce a non-intrusive technique that facilitates RefA to a degree that is adequate for touching all stored data within time intervals that are less than a target maximum cell retention time. This is achieved by selecting the appropriate application parameters while considering the performance overhead and quality loss that may be incurred.

- We realize our approach on a commodity server with a complete system stack and show for the first time the benefits of the RefA on a real system capturing the time-dependent system and application data interactions under relaxed DRAM refresh rates. The stack allows nondisruptive operation of the whole system under relaxed DRAM refresh rates, which enable us to evaluate the proposed method. 
- We apply the proposed approach on the Pochoir stencil compiler to expose parameters and allow us to control the scheduling of memory accesses and exploit the RefA.

- We evaluate the efficacy of our approach on the developed experimental platform using with a variety of stencilbased algorithms that are commonly used in numerical analysis, image processing and cellular automata.

Our findings demonstrate that it is possible to extend the refresh rate of $A R$ by orders of magnitude and even omit it with limited quality loss and performance overheads.

The rest of the paper is organized as follows. Section II describes the typical DRAM organization and refresh operation, while it discusses the related work and motivates the proposed work. Section III presents the proposed approach, while Section IV discusses its implementation. Section V describes the executed benchmarks and presents the evaluation results. Finally, conclusions are drawn in Section VI.

\section{DRAM BACKGROUND}

In this section, we briefly describe the DRAM organization and the refresh operation, along with the state-of-the-art.

\section{A. DRAM organization}

A main memory system based on DRAMs is organized hierarchically into channels, ranks, banks, rows and columns, as shown in Fig. 1. Each DRAM module (referred to as DIMM) usually has two ranks consisting of a number of twodimensional arrays of DRAM cells, the so called banks. Each DRAM cell is a storing element of DRAM and consists of a capacitor and an access transistor. Each access transistor connects its associated capacitor to a wire called a bitline and is controlled by a wire called wordline. Cells sharing a wordline form a row. Before a row can be activated, all bitlines in the bank must be precharged. The row's wordline is enabled by connecting all capacitors in that row to their respective bitlines. This causes charge to flow from the capacitor to the bitline. Finally, the sense amplifier connected to that bitline detects the voltage change and amplifies it, driving the bitline fully either to the power rail or to zero voltage.

\section{B. Retention Time and Refresh Operation}

The simple structure of the DRAM array and of each DRAM cell may allow high storage density, however is not capable of retaining the stored charge for a long period due to the inherent transistor's leakage current. Such leakage

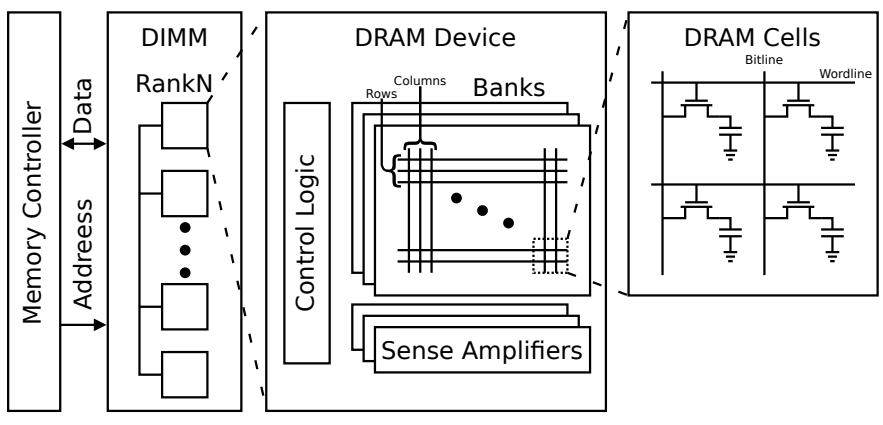

Fig. 1: DRAM memory system organization can eventually discharge the cell, manifesting a bit-flip. The duration that the cell can correctly retain its state (i.e. ' 0 ' or '1') without eventually experiencing any bit-flip is called retention time.

To avoid any error induced by the limited retention time, modern day DRAMs employ an Auto-Refresh mechanism that periodically recharges each cell in the DIMM by simply bringing the data from a row into the sense amplifiers and restoring them back in the row. To achieve this, the memory controller issues a refresh command every $t_{R E F I}$ cycles, at which point all DRAM banks simultaneously refresh a number of rows making the rank unavailable for $t_{R F C}$ cycles.

Currently, the refresh period $T_{R E F W}$, i.e. the interval within which all cells of the DIMM must be refreshed, is set according to the worst case retention time of all cells. In fact all DDRx technologies adopt today a $T_{R E F W}$ of $64 \mathrm{~ms}$ under nominal environmental conditions or $32 \mathrm{~ms}$ in case of temperatures higher than $85^{\circ} \mathrm{C}$.

Such a refresh period leads to considerable power and performance overheads, which are expected to worsen as the DRAM density increases [2]. In fact, the duration of the refresh operation increases linearly with each new DRAM generation, so the memory is expected to spend nearly one quarter of the time refreshing and one third of the power consumption for the refresh at 64Gb DRAM density [2].

\section{State-of-the-Art}

In an attempt to address the refresh related overheads, recent studies have shown that the retention time of cells varies considerably across and within a DRAM chip. Typically, only a very small number of cells needs to be refreshed once every $T_{R E F W}=64 \mathrm{~ms}$ [9], [10], [2], [11].

The so called multirate refresh techniques exploit such nonuniformity in retention time of DRAM cells to reduce the frequency of DRAM refresh. Such schemes [1], [3], [12], [13], [14], [15], [16] group rows into different bins based on the retention time profiling and apply a higher refresh rate only for rows belonging to the lower retention time bin. However, such approaches are highly intrusive since they assume fine grain control of the refresh rate, i.e. at the level of the row and have recently been deemed impractical since they neglect the fact that the retention time of each cell can change at runtime [17], [4].

Some recent works have tried to relax the refresh rate and address the resulting errors either by correcting them through traditional error-correcting codes [4] or by allowing them to happen and exploiting the error-resiliency of application for reducing their impact on quality [5], [6]. Although such techniques are interesting they have not yet systematically exploited the inherent refresh that takes place during each memory access which is the primary target of our proposed approach. The works in [8], [7] tried to exploit the implicit refreshes through DRAM accesses. However they require substantial changes in the DRAM controller. This makes them impractical since controllers are currently providing restricted access even to basic parameters (i.e. refresh-rate). Due to the 
intrusive required modifications such works have also been evaluated only on simulators and can not yet capture the real efficacy of the refresh by access mechanism. Refrint [18] is targeting eDRAM caches to optimize scheduling of writeback to memory based on the tracking of accesses, it is only feasible in caches as the size of caches is much smaller than that of main memory.

In this paper, we aim at systematically exploiting the RefA property, with no intrusive hardware modifications, and evaluating its efficacy on a commodity server with a complete system stack as we explain later.

\section{Proposed APPROACH}

Every DRAM access naturally opens the accessed row and consequently restores the leaked charge in the capacitor of DRAM cells, thus incurring an implicit refresh operation. Refresh-by-Access can be exploited to significantly relax the $A R$, while restricting the number of manifested errors.

To understand the proposed concept, let us consider a simple scenario as depicted in Fig. 2 and assume that a running application triggers $N_{r}$ Memory Accesses (MemA) to the $r$ address in memory so that:

$$
M e m A_{r}=\left\{M e m A_{0, r}, \ldots, M e m A_{N_{r}, r}\right\}
$$

Clearly, the intervals between consecutive accesses to the same address can be calculated as:

$$
\Delta t_{i, r}=t_{M e m A_{i+1, r}}-t_{M e m A_{i, r}}
$$

If the maximum of all $\Delta t_{i, r}$ is smaller than a target retention time $T_{\text {target }}$ of the DRAM cells, i.e.

$$
\max \left(\Delta t_{i, r}\right) \leq T_{\text {target }}
$$

then all cells would be implicitly refreshed though the memory accesses. In this case, we can relax the conventional $A R$ upto $T_{\text {target }}$ while maintaining the actual Bit-Error Rate (BER) low, since no cell that has up to $T_{\text {target }}$ retention time will fail. In fact, the BER will be bounded to a value lower than when we adopt $A R$ of $T_{\text {target }}$

$$
B E R\left(\max \left(\Delta t_{i, r}\right)\right) \leq B E R_{\text {target }}
$$

To demonstrate the potential of our approach, we have considered the following proof-of-concept. We used known test patterns [19] for characterizing the BER of 8GB DIMMs by aggressively relaxing the $A R$ from the conservative $64 \mathrm{~ms}$ to $1 \mathrm{sec}$ and up to $30 \mathrm{sec}$. The resulted Cumulative Distribution Function $(C D F)$ is depicted in Fig. 3.

For showing the efficacy of our scheme, we turn off the $A R$ and test the resulting manifested errors of an artificial benchmark that exploits RefA. The benchmark issues memory accesses required for touching all the stored data iteratively within intervals of $10 \mathrm{sec}$ and $20 \mathrm{sec}$. Essentially, we have ensured that $\max \left(\Delta t_{i, r}\right)=\{10,20\} \mathrm{sec}$ in each case.

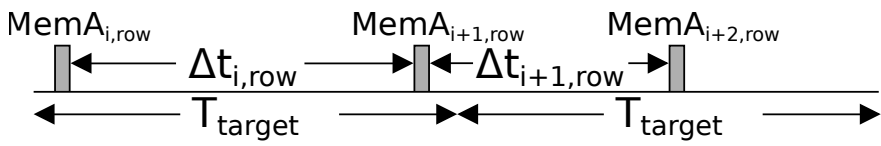

Fig. 2: Graphical representation of our approach where all time intervals between consecutive memory accesses are being kept lower than a target time interval (target retention time).

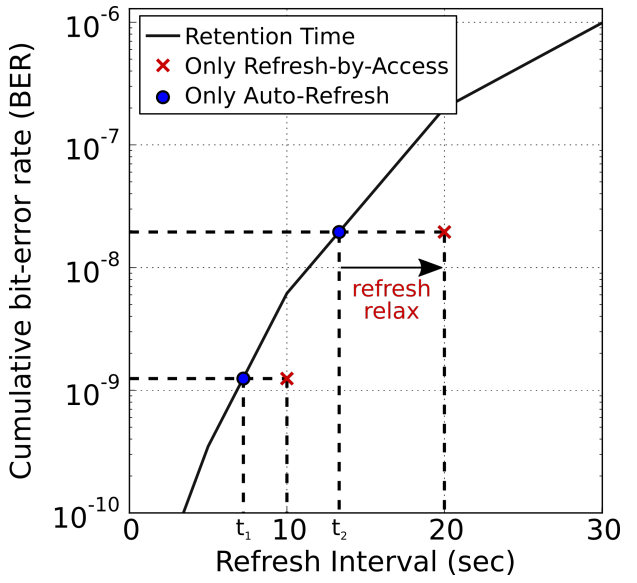

Fig. 3: Cumulative distribution function of bit-errors for relaxed refresh rate. The impact of RefA is also being showcased by showing the observed BER of a benchmark.

Interestingly, we can observe that by ensuring that all memory access intervals are bound to a value, then the resulting BER is equal to the one achieved by using $A R$ with a more frequent refresh. In particular, the first case resulted in a BER of $10^{-9}$ that is equal to the one achieved with $A R$ of $8.6 \mathrm{sec}$ while the second case resulted in a BER of $2 * 10^{-8}$ that is equivalent to AR of $12 \mathrm{sec}$.

Our suggestion is that if we schedule the memory accesses incurred by an application in such a way that all rows are iteratively touched within a target refresh interval (that is adequate for keeping the BER at acceptable levels), then the refresh rate of AR can be aggressively relaxed, in our case be omitted. The challenge that we need to address is how could we achieve the above target, which brings us to the details of the proposed methodology that is described next.

\section{Methodology}

The main attribute that we need to control for relaxation of the $A R$ operations is memory accesses $(M e m A)$, which are application-dependent. By setting suitably such parameters, we can schedule the memory accesses in such way that we eventually meet the required conditions of Equation 3.

Fig. 4 shows our methodology to achieve this. Initially, we are selecting a set of values for the application parameters $p=\left\{p_{1}, p_{2}, \ldots\right\}$, such as data structure size, scheduling of the

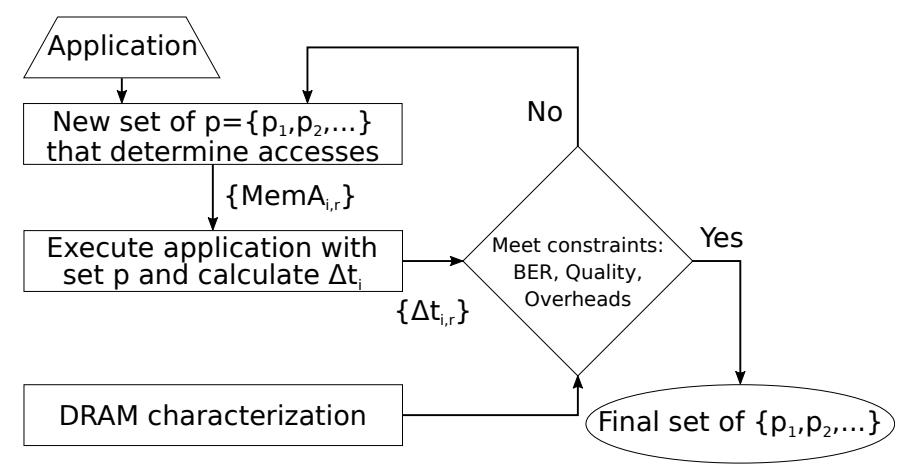

Fig. 4: Block diagram of the steps to explore the design space of an application. 
accesses or other parameters that are determining the accesses of the application $M e m A_{i, r}$. We are executing the application with the selected parameter set. At the same time we are calculating the $\Delta t_{i, r}: \forall i, r$ of the run, the overheads that are introduced for this set of parameters or any quality metric of interest.

Based on the DRAM characterization, we can calculate the $B E R\left(\max \left(\Delta t_{i, r}\right)\right)$. If the achieved $B E R$ is smaller than a selected $B E R_{\text {target }}$ and all the other constraints that the designer has specified (e.g. limited performance overhead) are satisfied then we conclude that this set of parameters is acceptable. In case that one of the constraints is violated, we iterate the process with a new set of parameters until all constraints meet.

Such a methodology can be well applied to iterative algorithms, as they regularly access the same data. In this work, we are going to focus on stencil-based algorithms to demonstrate our method.

\section{Case Study: Stencil-Based Algorithms}

Stencil-based algorithms are a class of iterative kernels. In each sweep, the stencil updates all the elements of a n-dimensional grid using neighboring elements in a fixed pattern, called stencil.

We are using Pochoir stencil compiler [20], which is built on top of Cilk Plus multithread extension and uses trapezoidal decompositions, which utilize the cache efficiently. The decomposition is breaking down the problem into smaller tasks that are responsible for a part of the grid and the associated stencil for a number of sweeps. It decomposes the sweep domain when the number of sweeps is greater than a threshold and recursively process the lower sub-trapezoids before the upper ones or the base domain. We are modifying the compiler so that it schedules the memory accesses to facilitate RefA.

For our method to work, we need to control the interval between consecutive memory accesses. For this reason, we are introducing barriers, we call those borders, every border ${ }_{\text {height }}$ sweeps. Borders break down the sweep domain on the same sweep for all the grid, as shown on Fig. 5 with the dashed line. The border essentially does not allow to start any consequent sweep forcing all tasks in the current border height $_{\text {to com- }}$ plete. We can measure the time intervals between consecutive

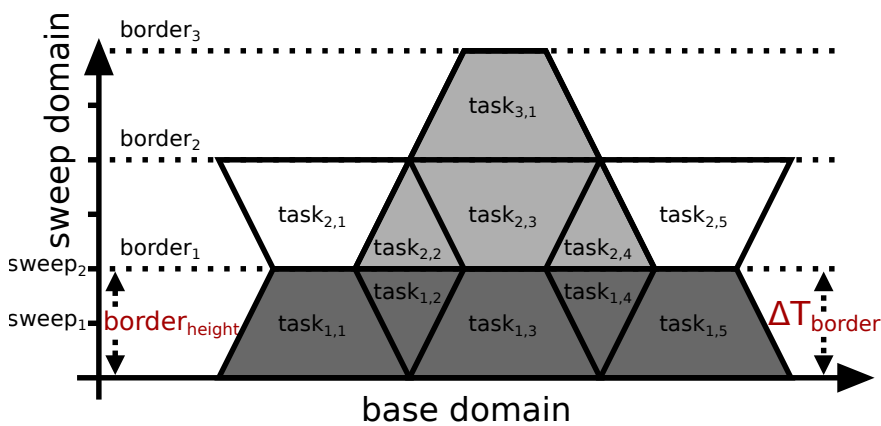

Fig. 5: Trapezoidal decomposition of 1-D problem. All the tasks below border 1 must be completed in order to continue to next sweep. completions of borders, we call this interval $\Delta t_{\text {border. All }}$ elements will be accessed at least once during $\Delta t_{\text {border }}$, we can conclude that each $\Delta t_{i, r}$ will be bound to be less than $\Delta t_{\text {border }}$. Fig. 5 shows also the Original implementation that would opportunistically progress as many sweeps as possible, specifically after finishing $\operatorname{task}_{1,1}, \operatorname{tas}_{1,2}$ and $t a s k_{1,3}$, it will compute all the tasks up to the task $k_{3,1}$, highlighted in gray.

We are setting border height as a parameter for controlling the $\Delta t_{\text {border }}$. Lowering the border $r_{\text {height }}$ will reduce the required computation of each task also the $\Delta t_{\text {border }}$, while increasing the number of tasks. The border height must be carefully selected so that the BER resulted from the $\Delta t_{\text {border }}$ will be kept under the set threshold, $B E R_{\text {target }}$. Furthermore, tasks should not be broken down to very small ones as there is a considerable performance overhead caused by the function calls and by breaking the cache efficiency.

\section{Evaluation}

In this section, we describe our experimental setup and analyze our results.

\section{A. Applications}

To evaluate our approach, we have applied it to Pochoir compiler and used it for a range of stencil-based algorithms, that have different number of dimensions, grid size, data size of each element and complexity of the stencil. Specifically, we have experimented with the algorithms of Heat Dissipation (Heat) [21], Conways's Game of Life (Life) [21] and an artificial benchmark (Artf), the characteristics of which are shown in Table 1. The Artf is designed as a $3 \times 3$ kernel on 2-dimensional grid which in each sweep applies binary arithmetic and shifting between different elements so that each bit-error will persist during the execution and be manifested once at the end result.

\section{B. Experimental Setup}

One of the aims of our work is to evaluate the efficacy of our method on a real system in order to capture the time dependent system and application memory access interactions, as opposed to existing works that were evaluated on simulators. To this end, we have built an experimental platform based on a dual-socket commodity server. Each socket hosts an Intel ${ }^{\circledR}$ Xeon E5-2650 (Sandy Bridge) processor featuring an integrated memory controller (iMC) to control the DRAM devices attached to the socket, specifically four 8 GB DDR3 DIMMs at $1600 \mathrm{MHz}$. The iMC exposes a set of configuration registers [22] to enable or disable refresh for the entire DRAM.

Following the fact that each iMC controls a single memory domain of the socket, DRAMs attached to different CPU

\begin{tabular}{|c|c|c|c|c|c|c|}
\hline \multirow{2}{*}{ Benchmark } & \multicolumn{2}{|c|}{ Grid Size } & \multicolumn{2}{c|}{ Memory (GB) } & \multicolumn{2}{c|}{ Execution Time } \\
\cline { 2 - 7 } & $\min$ & $\max$ & $\min$ & $\max$ & $\min$ & $\max$ \\
\hline \hline Heat-2D & 18000 & 30000 & 9.3 & 24 & $38 \mathrm{~s}$ & $96 \mathrm{~s}$ \\
Heat-3D & 780 & 1020 & 7.4 & 16.6 & $56 \mathrm{~s}$ & $263 \mathrm{~s}$ \\
Life-2D & 18000 & 30000 & 1.5 & 4 & $46 \mathrm{~s}$ & $156 \mathrm{~s}$ \\
Artf-2D & 18000 & 30000 & 6.2 & 16 & $37 \mathrm{~s}$ & $98 \mathrm{~s}$ \\
\hline
\end{tabular}

Table 1: Benchmarks 


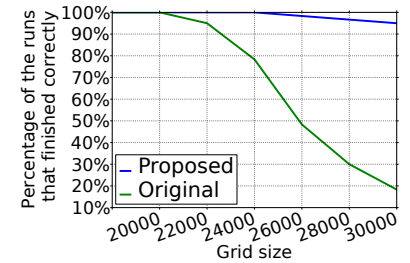

(a) Percentage of the runs that completed correctly

Fig. 6: Artf benchmark

sockets can have $A R$ enabled or disabled separately. In our dual-socket system, the first memory domain is deemed reliable with the nominal $A R$ applied, in which the Operating System is allocated, while the second one is configured with no refresh. In this way, we can safe guard the kernel data that cannot tolerate errors in the reliable domain for avoiding any catastrophic failures and run the application on the second memory domain.

\section{Quality Metric}

The algorithms, that we are experimenting with are not resilient to errors. Even one error can have catastrophic results as it may propagate to neighboring elements. So the quality metric that we measure is the percentage of runs that completed correctly. For further characterization and to be able to measure the number of bit-errors, we are using the Artf.

\section{Experimental Results}

1) Artificial Benchmark: We start with the results obtained from Artf as it is possible to measure manifested errors. We are comparing the benchmark compiled with the Original implementation of the Pochoir and our Proposed one.

In our experiments, we sweep the border height from 10 to 40 and the grid size from 18000 to 30000 . The border height in the results of Fig. 6 is chosen to be 20 so that the following constraints are met: $i$ ) the maximum introduced performance overhead is lower than $15 \%$ and ii) the maximum $\Delta t_{\text {border }}$ is below 2 seconds that corresponds to BER of $10^{-10}$. The results are obtained after executing Artf 1340 times, until results stabilize, while varying the grid size. Fig. 6a compares the percentage of the correct runs of the two implementations and Fig. $6 \mathrm{~b}$ shows the average number of bit-errors occurred. We can observe that given the constraints, our Proposed implementation outperforms retaining a high percentage, over $95 \%$, of correct runs across the range of grid sizes, while the Original implementation rapidly decrease its quality.

2) Application Benchmarks: Fig. 7 and 8 present the design space exploration for the performance overhead and for the $\Delta t_{\text {border }}$ of the benchmarks Heat-2D and Heat-3D. The design space is populated from the results of runs with variable border height and grid size. We are sweeping the border height for Heat-2D from 10 to 40 and for Heat-3D from 2 to 10 . Respectively, we are sweeping the grid size from 18000 to 30000 and from 900 to 1020 .

We observe that the performance overhead is mainly affected by the border height caused by the disruption of

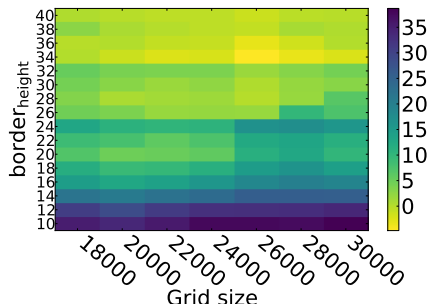

(a) Heat-2D

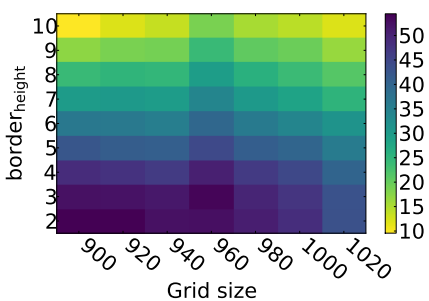

(b) Heat-3D
Fig. 7: Percentage of performance overhead introduced from our technique

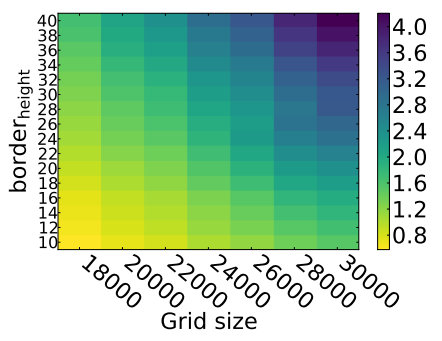

(a) Heat-2D

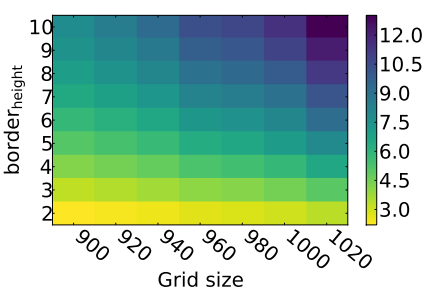

(b) Heat-3D
Fig. 8: Achieved $\Delta t_{\text {border }}$ in seconds

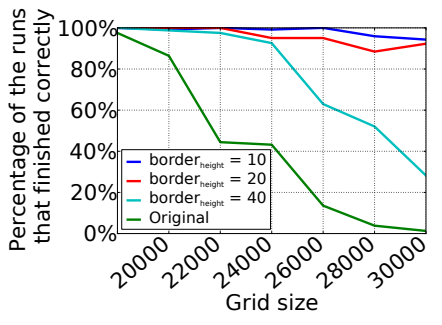

(a) Heat-2D

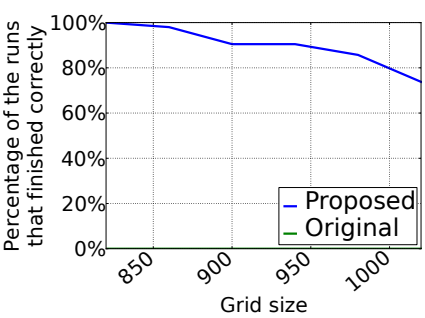

(b) Heat-3D
Fig. 9: Percentage of the runs that completed correctly

cache locality and by the increased number of function calls while having smaller tasks. $\Delta t_{\text {border }}$ is affected by both the border $_{\text {height }}$ and the grid size, as the border height affects the number of stencil computations for each task and the grid size affects the number of tasks that belong in each border height.

We are using the design space to select the parameter values that minimize refresh operations given different constraints such as maximum acceptable performance overhead and the maximum $\Delta t_{\text {border }}$.

Based on the performed design space exploration, we choose to analyze 3 possible scenarios for better understanding the efficacy of our approach. We are selecting parameters to optimize: $i$ ) for the minimum $\Delta t_{\text {border }}$ and consequently the lowest $B E R$, ii) for the least performance overhead and iii) for both those two previous criterion together. So we are exploring the error characteristics for the 3 scenarios with border height $= \begin{cases}10,40,20 \\ \text {, }\end{cases}$ equivalent $\max \left(\Delta t_{\text {border }}\right)=\{1.5,4.5,2.4\}$ seconds and $\max ($ overhead $)=\{38 \%, 5 \%, 12 \%\}$. We can see the results of 1968 runs in Fig. 9a, which depicts the percentage of the runs that completed correctly.

We can observe that for the first and third scenario, the percentage of correct runs remain over $90 \%$ for all the grid sizes, while for border $r_{h e i g h t}=40$, the percentage decreases 


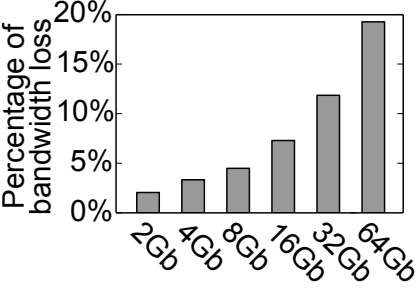

Device density

(a) Throughput loss due to refresh

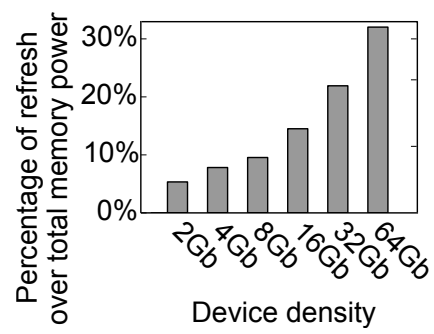

(b) Power overhead of refresh operations
Fig. 10: Refresh implications on power and performance on current and future DRAM technologies [1]

more rapidly. However in each case the results are better than the Original implementation.

In Fig. 9b, we explore one setting for Heat-3D with parameter of border $r_{\text {height }}=6$ with equivalent $\max \left(\Delta t_{\text {border }}\right)=8$ seconds and $\max ($ overhead $)=44 \%$. For the selected range of grid sizes, the original implementation has no devastating results even in the smaller grids, while the quality degrades slowly with our Proposed implementation.

\section{E. Performance and Power Gains}

Based on the above results it is evident that there are cases where our approach can relax or omit refresh with minor quality losses. Currently, we do not consider the cost of recovery, however exascale systems [23], [24] do already implement efficient recovery techniques. If we omit the $A R$, we obviously help to limit the $A R$ overheads. As seen on Fig. 10, we can gain from $4.4 \%$ performance in current technologies and up to $19 \%$ in future technologies and decrease the power consumption of the memory system by $9.6 \%$ to $31 \%$.

Since our technique can help relax the $A R$ or even disable it by merely scheduling the accesses of the application, RefA can work orthogonally and complementary to other techniques that relax the refresh rate.

\section{CONCLUSIONS}

In this paper, we present a non-intrusive method for relaxing the refresh-rate by systematically exploiting the implicit refresh that take place during each memory access. The proposed approach, identifies the suitable algorithmic parameters that influence the access patterns and sets them such that all stored data are being accessed within a target time interval without incurring major performance penalty. By ensuring that all accesses take place within the target time interval we guarantee than the bit-error-rate is kept within acceptable levels. The proposed approach not only helps to limit the power overhead by significantly relaxing the refresh-rate with no required hardware modification but also helps to alleviate the performance penalties incurred by the refresh, The efficacy of the proposed method is demonstrated on an off-the-shelf server running a fully fledged Linux OS and when applied to stencil-based algorithms results show that it is even possible to completely disable DRAM refresh with minor quality loss.

\section{ACKNOWLEDGEMENTS}

The presented research effort has received funding from the European Community' Horizon 2020 programme under grant no. 688540 (UniServer).

\section{REFERENCES}

[1] J. Liu, B. Jaiyen, R. Veras, and O. Mutlu, "RAIDR: Retention-aware intelligent dram refresh," in Proceedings of the 39th ISCA '12, pp. 112

[2] J. Liu, B. Jaiyen, Y. Kim, C. Wilkerson, O. Mutlu, J. Liu, B. Jaiyen, Y. Kim, C. Wilkerson, and O. Mutlu, "An experimental study of data retention behavior in modern DRAM devices," in Proceedings of the 40th ISCA'13, p. 60.

[3] R. Venkatesan, S. Herr, and E. Rotenberg, "Retention-Aware Placement in DRAM (RAPID): Software Methods for Quasi-Non-Volatile DRAM," in 12th International Symposium on HPCA '06, pp. 157-167.

[4] M. K. Qureshi, D.-H. Kim, S. Khan, P. J. Nair, and O. Mutlu, "Avatar: A variable-retention-time (vrt) aware refresh for dram systems," in Proceedings of the 45th IEEE DSN'15, pp. 427-437.

[5] S. Liu, K. Pattabiraman, T. Moscibroda, and B. G. Zorn, "Flikker: Saving dram refresh-power through critical data partitioning," in Proceedings of the 16th ASPLOS '11, pp. 213-224.

[6] A. Raha, H. Jayakumar, S. Sutar, and V. Raghunathan, "Quality-aware data allocation in approximate DRAM?" in International Conference on CASES'15, pp. 89-98.

[7] H. Hassan, G. Pekhimenko, N. Vijaykumar, V. Seshadri, D. Lee, O. Ergin, and O. Mutlu, "Chargecache: Reducing dram latency by exploiting row access locality," IEEE Symposium on HPCA '16, pp. 581-593.

[8] M. Ghosh and H. H. S. Lee, "Smart refresh: An enhanced memory controller design for reducing energy in conventional and 3d die-stacked drams," in 40th Annual IEEE/ACM MICRO '07, pp. 134-145.

[9] I. Bhati, M. T. Chang, Z. Chishti, S. L. Lu, and B. Jacob, "DRAM Refresh Mechanisms, Penalties, and Trade-Offs," IEEE Transactions on Computers, vol. 65, no. 1, pp. 108-121, jan 2016.

[10] K. Kinam Kim and J. Jooyoung Lee, "A New Investigation of Data Retention Time in Truly Nanoscaled DRAMs," IEEE Electron Device Letters, vol. 30, 2009.

[11] M. Jung, D. M. Mathew, C. Weis, and N. Wehn, "Invited - approximate computing with partially unreliable dynamic random access memory approximate dram," in Proceedings of the 53rd DAC '16, pp. 101-104.

[12] I. Bhati, Z. Chishti, S.-L. Lu, B. Jacob, I. Bhati, Z. Chishti, S.-L. $\mathrm{Lu}$, and B. Jacob, "Flexible auto-refresh," ACM SIGARCH Computer Architecture News, vol. 43, no. 3, pp. 235-246, jun 2015.

[13] Z. Cui, S. A. McKee, Z. Zha, Y. Bao, and M. Chen, "DTail," in Proceedings of the 28th ACM ICS'14, pp. 43-52.

[14] M. Jung, D. M. Mathew, C. Weis, and N. Wehn, "Efficient reliability management in SoCs - an approximate DRAM perspective," in 21st ASP-DAC '16, pp. 390-394.

[15] J. Mukundan, H. Hunter, K.-h. Kim, J. Stuecheli, and J. F. Martínez, "Understanding and mitigating refresh overheads in high-density DDR4 DRAM systems,' in 40th ISCA '13.

[16] P. J. Nair, C.-C. Chou, and M. K. Qureshi, "Refresh pausing in DRAM memory systems," ACM TACO '14, vol. 11, no. 1, pp. 1-26.

[17] Y. Han, Y. Wang, H. Li, and X. Li, "Data-aware dram refresh to squeeze the margin of retention time in hybrid memory cube," in IEEE/ACM ICCAD' 14

[18] A. Agrawal, P. Jain, A. Ansari, and J. Torrellas, "Refrint: Intelligent refresh to minimize power in on-chip multiprocessor cache hierarchies," in 19th IEEE International Symposium on HPCA '13, pp. 400-411.

[19] C. Huzum and P. Cascaval, "March test for static neighborhood patternsensitive faults in random-access memories," Elektronika ir Elektrotechnika' 12 .

[20] Y. Tang, R. A. Chowdhury, B. C. Kuszmaul, C.-K. Luk, and C. E. Leiserson, "The pochoir stencil compiler," in 23rd ACM SPAA '11, p. 117.

[21] J. F. Epperson, An Introduction to Numerical Methods and Analysis. Wiley-Interscience, 2007.

[22] "Intel Xeon Processor E5-1600/2400/2600/4600 (E5-Product Family) Product Families Datasheet, Volume Two," 2012. [Online]. Available: http://www.intel.com/content/www/us/en/processors/xeon/xeone5-1600-2600-vol-2-datasheet.html

[23] M. Gamell, D. S. Katz, H. Kolla, J. Chen, S. Klasky, and M. Parashar, "Exploring automatic, online failure recovery for scientific applications at extreme scales," in Proceedings of SC '14, pp. 895-906.

[24] J. T. Daly, "A higher order estimate of the optimum checkpoint interval for restart dumps," Future Generation Computer Systems, vol. 22, no. 3, pp. 303-312, Feb. 2006. 